Abstract

\title{
Entropy, Statistical Thermodynamics and Stochastic Processes ${ }^{+}$
}

\section{Themis Matsoukas}

Pennsylvania State University, State College, PA, USA

+ Presented at the Entropy 2021: The Scientific Tool of the 21st Century, 5-7 May 2021; Available online: https://sciforum.net/conference/Entropy2021/.

Published: 5 May 2021

The undeniable appeal of statistical mechanics has led to numerous attempts to extend its tools to processes and problems outside the realm of molecules and physical particles. However, no formal theory exists to guide us on the application of statistical mechanics outside physics and chemistry. In this talk I will show that the basic elements of statistical mechanics are universal to generic stochastic processes.

The theory views a stochastic process as a network of chemical reactions. We begin with a finite sample of the the event space at time zero and construct all possible future paths based on the transformations that are possible under the rules of the stochastic process. We define the ensemble of states that can be reached in a fixed number of steps from the initial state (feasible space), define its probability and formulate its master equation. We show that when the size of the initial sample increases indefinitely (asymptotic limit), the feasible space becomes continuous but its probability distribution converges to discrete points that represent thermodynamic phases. If only one phase is present the ensemble is represented by its most probable distribution. We work out the calculus of the most probable distribution in the asymptotic limit, identify the functional whose maximization produces that distribution and express the most probable distribution in terms of a partition function and its derivatives. We analyze four problems under this theory: (a) random walk, (b) binary clustering (c) binary fragmentation and (d) equilibrium exchange, and give examples of phase splitting in these systems.

(C) 2021 by the author. Licensee MDPI, Basel, Switzerland. This article is an open access article distributed under the terms and conditions of the Creative Commons Attribution (CC BY) license (http://creativecommons.org/licenses/by/4.0/). 